\title{
Revue de la littérature
}

\section{Plasmocytome osseux solitaire de la mandibule : un cas clinique et revue de la littérature}

\author{
Hicham Sabani ${ }^{1}{ }^{,}{ }^{*}$, Kamal Fiqhi ${ }^{1}$, Adil Arrob ${ }^{1}$, Karim El Khatib ${ }^{1}$ \\ ${ }^{1}$ Service de chirurgie maxillo-faciale, chirurgie plastique et stomatologie. Hôpital militaire d'instruction Mohammed V, \\ avenue des Forces Armées Royales, Rabat, Maroc
}

(Reçu le 18 juin 2015, accepté le 2 juillet 2015)

Mots clés : plasmocytome / solitaire / mandibule / radiothérapie
Key words:

Plasmacytoma / solitary / mandible / radiotherapy
Résumé - Introduction : Le plasmocytome osseux solitaire est une tumeur maligne qui se caractérise par une prolifération de cellules plasmatiques monoclonales. Il atteint préférentiellement le squelette axial notamment le rachis et le pelvis. Sa localisation mandibulaire est exceptionnelle. Observation : Un homme de 49 ans venait consulter pour une tumeur mandibulaire droite évoluant depuis six mois de façon asymptomatique. Après une exérèse chirurgicale à visée diagnostique réalisée sous anesthésie générale, l'examen histologique montrait qu'il s'agissait d'un plasmocytome. Le bilan d'extension réalisé permettait de confirmer le diagnostic de plasmocytome osseux solitaire de la mandibule. Le patient bénéficiait d'une radiothérapie externe adjuvante et l'évolution était favorable. Discussion : À partir de cette observation clinique et d'une revue de la littérature, les aspects cliniques, radiologiques, thérapeutiques et évolutifs de cette pathologie rare sont discutés. La localisation mandibulaire du plasmocytome osseux est exceptionnelle et le diagnostic est avant tout histologique.

\begin{abstract}
Solitary bone plasmacytoma of the mandible: a case report and review of literature. Introduction: A solitary plasmacytoma is a malignant tumor characterized by proliferation of monoclonal plasma cells. It preferentially reaches the axial skeleton, in particular the rachis and pelvis. Its location in the jaw is exceptional. Observation: A 49-year-old male patient consulted for a right mandibular tumor which had evolved for six months in an asymptomatic way. After surgical excision performed under general anesthesia in order to establish a diagnosis, histological examination showed that it was a plasmacytoma. The extension work-up confirmed the diagnosis of solitary bone plasmacytoma of the mandible. The patient received adjuvant external radiotherapy. The outcome was favorable. Discussion: From this clinical observation and the review of the literature, the clinical aspect, radiological signs, therapeutic solutions and outcome of this rare disease are discussed. Mandibular location of bone plasmacytoma is very rare and diagnosis is first histological.
\end{abstract}

\section{Introduction}

Le plasmocytome osseux solitaire (POS) est un processus tumoral malin dérivant d'un clone unique de lymphocytes $B$ plus ou moins différenciés. L'absence de sécrétion d'immunoglobulines (Ig) monoclonales et de dissémination de la prolifération définissent le caractère solitaire du POS. La localisation mandibulaire de cette tumeur est exceptionnelle.

À partir d'une observation clinique et d'une revue de la littérature, les aspects cliniques, radiologiques, thérapeutiques et évolutifs de cette pathologie rare sont discutés.

\section{Observation}

Un patient de 49 ans, sans antécédent pathologique notable, était venu consulter le 6 décembre 2011 au service de chirurgie maxillo-faciale, chirurgie plastique et stomatologie de l'hôpital militaire Mohammed V de Rabat. Il était adressé par un médecin généraliste pour une tuméfaction de l'angle mandibulaire droit évoluant depuis six mois, de façon asymptomatique.

L'examen exobuccal révélait une masse arrondie, faisant $5 \mathrm{~cm}$ de diamètre, de consistance dure, indolore, faisant corps avec l'os. Le signe de Vincent était négatif.

\footnotetext{
*Correspondance : hichamsabani@gmail.com
}

This is an Open Access article distributed under the terms of the Creative Commons Attribution License (http://creativecommons.org/licenses/by/4.0), which permits unrestricted use, distribution, and reproduction in any medium, provided the original work is properly cited 
L'examen endobuccal montrait une légère limitation de l'ouverture buccale de $35 \mathrm{~mm}$. La masse tumorale revêtue d'une muqueuse d'allure normale s'étendait de la dent 45 jusqu'au trigone rétromolaire en comblant le vestibule inférieur droit. Une édentation du secteur molaire inférieur droit était notée. Les aires ganglionnaires cervico-faciales étaient libres.

L'orthopantomogramme objectivait une image lytique mono-géodique de contours réguliers de l'angle mandibulaire droit plongeant dans le ramus, faisant évoquer un améloblastome ou un fibrome améloblastique (Fig. 1).

La tomodensitométrie maxillo-faciale montrait un processus ostéolytique centré sur l'angle mandibulaire droit, à double composante tissulaire et kystique, avec rupture des corticales par endroits mais sans extension aux parties molles (Fig. 2 et 3).

Le 12 décembre 2011, le patient bénéficiait sous anesthésie générale d'une exérèse chirurgicale totale de la masse tumorale à visée diagnostique. Il s'agissait en fait d'une lésion kystique dont la membrane était épaisse, d'aspect grisâtre et de structure friable. L'examen anatomopathologique de la pièce opératoire montrait un aspect morphologique et un profil immunohistochimique compatible avec un plasmocytome à chaîne kappa.

Le bilan d'exploration était ensuite orienté par la suspicion de myélome multiple devant la lyse osseuse et les chiffres élevés de la vitesse de sédimentation ( $1^{\text {re }}$ heure : $35 \mathrm{~mm}$, $2^{\mathrm{e}}$ heure : $50 \mathrm{~mm}$ ). Ce bilan était composé de :

- une numération formule sanguine ;

- un ionogramme sanguin avec fonction rénale ;

- un bilan phosphocalcique ;

- un myélogramme ;

- une scintigraphie osseuse.

Ce bilan était normal permettant ainsi d'éliminer le diagnostic de myélome multiple et de confirmer le diagnostic de POS de la mandibule.

Par la suite, le patient était adressé au service de radiothérapie du même hôpital où il bénéficiait d'une radiothérapie externe adjuvante à la dose de $\mathbf{4 6}$ grays sur le lit tumoral pendant un mois.

Un suivi post-thérapeutique était instauré ; le patient était vu en consultation tous les trois mois au cours de la première année, puis tous les six mois au cours de la deuxième et de la troisième année. Lors de chaque consultation, le contrôle était à la fois clinique, radiologique (orthopantomogramme) et biologique (VS, immunoélectrophorèse des protides et myélogramme). Après un recul de 36 mois, le patient ne présentait aucune rechute et le contrôle locorégional était bon (Fig. 4).

\section{Discussion}

Les hémopathies plasmocytaires représentent 1 à $2 \%$ de l'ensemble des néoplasies humaines [1]. Parmi les tumeurs

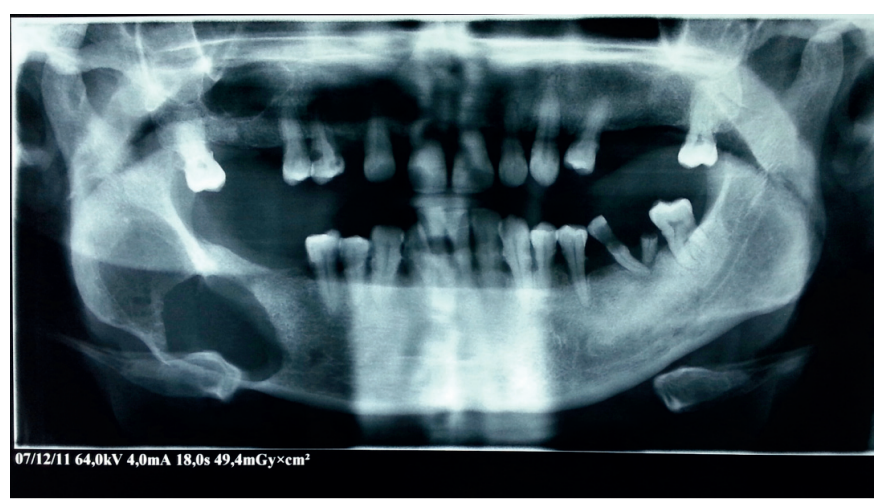

Fig. 1. Orthopantomogramme montrant une image ostéolytique monogéodique de contours réguliers au niveau de l'angle mandibulaire droit.

Fig. 1. Orthopantomogram showing an osteolytic monogeodic image with regular contours at the right mandibular angle.

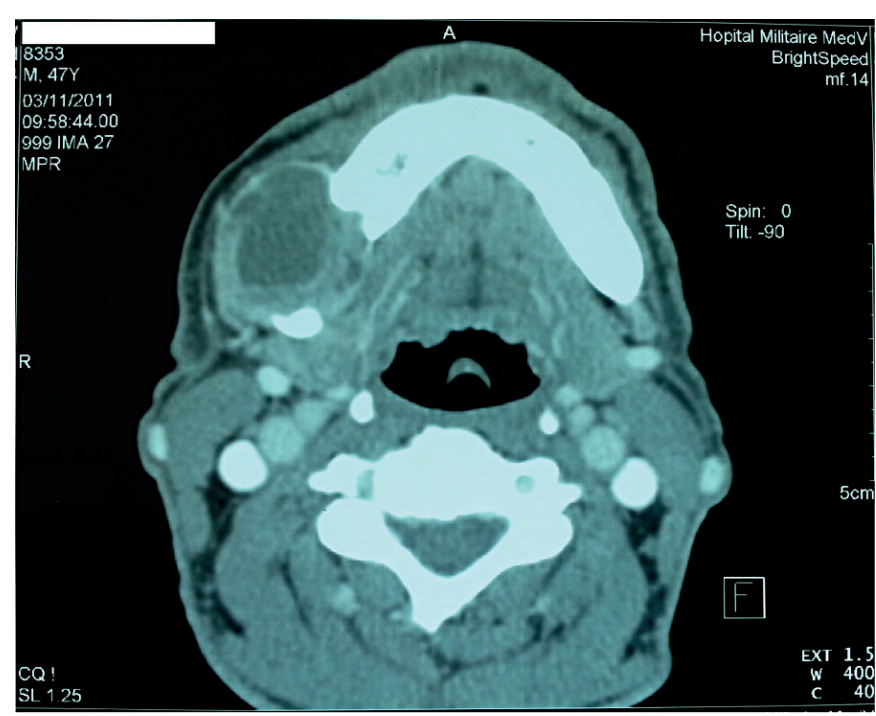

Fig. 2. Examen tomodensitométrique (coupe axiale) montrant un processus à double composante tissulaire et kystique lysant les deux corticales de l'angle mandibulaire droit.

Fig. 2. CT-scan (axial section) showing a dual process tissue and cystic component destroying both cortices of the right mandibular angle.

plasmocytaires, on distingue le myélome multiple, le plasmocytome extra-médullaire et le plasmocytome osseux solitaire (POS). Le myélome multiple représente $93 \%$ des tumeurs plasmocytaires [2] alors que le POS est plus rare et ne représente que $4 \%$ de ces tumeurs. Il est défini comme une lésion focale composée de plasmocytes malins mais sans envahissement médullaire diffus, ce qui le distingue du myélome multiple. Il touche préférentiellement le rachis et les os longs [3]. Les localisations crâniennes et maxillaires représentent $4 \%$ des 


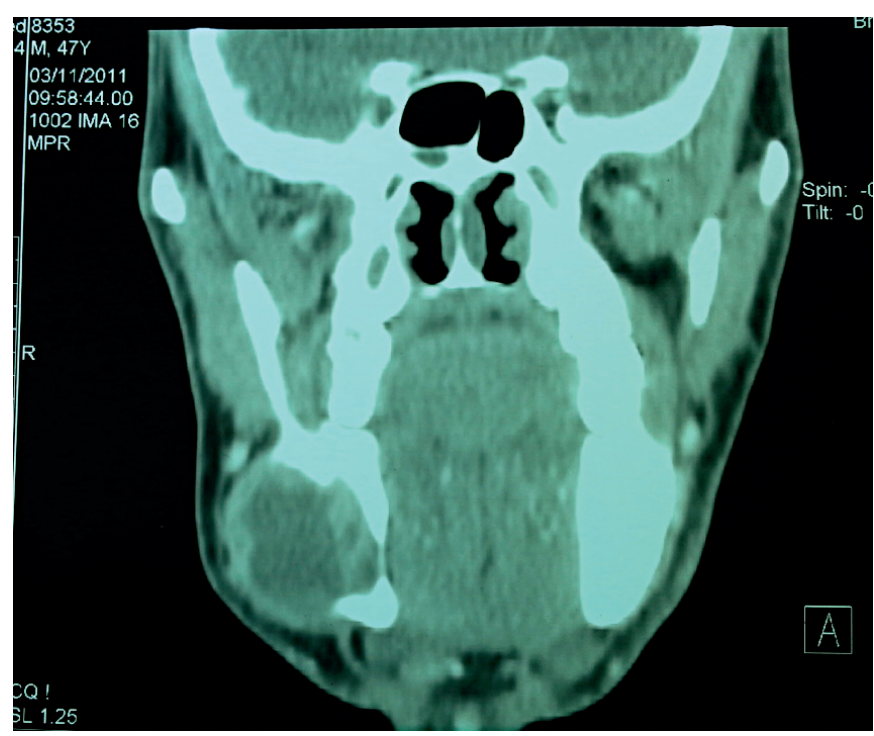

Fig. 3. Examen tomodensitométrique (coupe coronale) montrant un processus à double composante tissulaire et kystique lysant les deux corticales de l'angle mandibulaire droit.

Fig. 3. CT-scan (coronal section) showing a process double tissue and cystic component lysing both cortices of the right mandibular angle.

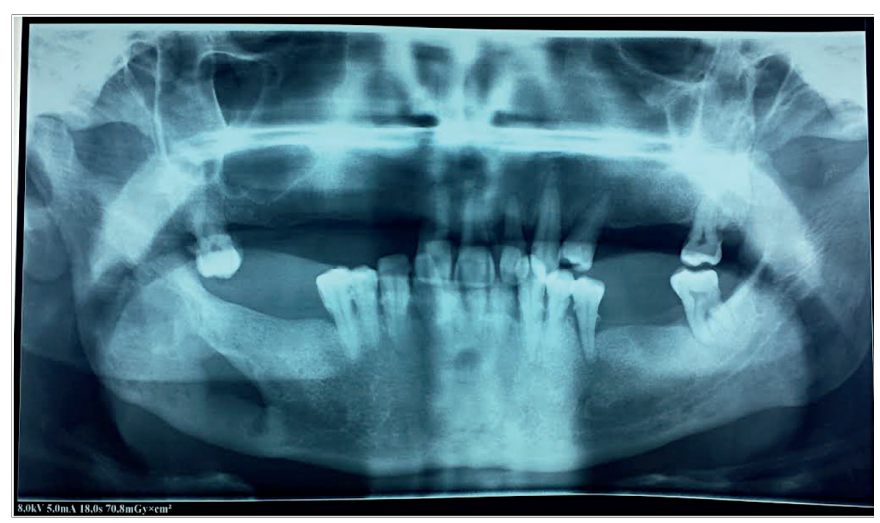

Fig. 4. Orthopantomogramme de contrôle (24 mois après chirurgie et radiothérapie) montrant une ossification progressive au niveau du site tumoral.

Fig. 4. Control orthopantomogram (24 months after surgery and radiotherapy) showing progressive ossification at the tumor site.

cas [4]. La mandibule est plus fréquemment atteinte que le maxillaire [5], particulièrement au niveau de la région postérieure qui est riche en cellules hématopoïétiques [6]. L'atteinte masculine est prédominante [7] et l'âge moyen de survenue est de 50 ans [5].

La symptomatologie clinique du POS de la mandibule n'est pas spécifique. Plusieurs signes et symptômes peuvent être observés isolément ou associés en fonction du stade évolutif de la tumeur et de sa localisation. La tuméfaction faciale, la mobilité dentaire et les troubles sensitifs sont les signes cliniques les plus fréquents. Rarement il peut être découvert à l'occasion d'une fracture pathologique, d'un saignement postavulsionnel dentaire ou d'un trouble de l'articulé dentaire pour les localisations condyliennes [6].

L'aspect radiologique est celui d'une image ostéolytique pouvant être mono- ou multiloculaire [8]. Les lésions radioopaques sont exceptionnelles [9]. La tomodensitométrie permet d'affiner l'image radiologique et d'étudier l'extension vers les corticales et les parties molles adjacentes. Ces aspects radiocliniques du POS de la mandibule posent le problème de diagnostic différentiel avec les tumeurs kystiques et pseudokystiques ainsi que les métastases des tumeurs malignes [8]. Le diagnostic de certitude du POS repose sur l'examen anatomopathologique complété par l'immunohistochimie [10].

Un certain nombre de critères sont nécessaires pour affirmer le caractère solitaire du POS et éliminer un myélome multiple $[11,12]$ :

- absence d'anémie ;

- bilan phosphocalcique normal;

- fonction rénale normale ;

- cytologie médullaire normale dans au moins deux sites différents ;

- absence d'autres lésions sur les radiographies du squelette ou sur la scintigraphie osseuse.

Le principal risque évolutif du POS est la survenue d'un myélome multiple avec des délais variables allant de quelques mois à dix ans [13]. La fréquence de cette transformation myélomateuse varie entre 45 et $75 \%$ [14].

Sur le plan thérapeutique, la radiothérapie constitue le traitement de référence du POS, soit exclusivement, soit après une chirurgie à visée essentiellement diagnostique. La radiothérapie, utilisée à des doses de 40 à $50 \mathrm{~Gy}(1,8$ à $2 \mathrm{~Gy} / \mathrm{j})$, permet d'obtenir un taux de contrôle local de plus de $90 \%$ avec également un effet antalgique $[1,15]$. L'adjonction d'une chimiothérapie n'est pas recommandée, celle-ci n'ayant pas prouvé son efficacité sur le taux de récidive ou d'évolution vers un myélome multiple [11].

Une seule étude prospective a rapporté un bénéfice avec l'association chimiothérapie et radiothérapie par rapport à la radiothérapie seule [16]. Dans cette étude, les auteurs ont évalué l'efficacité et la toxicité d'un protocole de chimiothérapie adjuvante avec de faibles doses de melphalan et de prednisone administrées aux patients atteints de POS après la radiothérapie. Au terme de cette étude, les auteurs ont estimé que l'utilisation de la chimiothérapie adjuvante après administration de doses suffisantes de radiothérapie chez les patients atteints de POS améliore la durée de la rémission et la survie globale. Cependant, les auteurs de cette étude avouent que le groupe d'essai était trop petit pour tirer des conclusions définitives [16]. 
Les facteurs prédictifs de la récidive locale semblent être : les doses insuffisantes de radiothérapie et la taille tumorale [17].

Après traitement du POS de la mandibule, la surveillance doit donc être minutieuse et durable. L'examen clinique, l'orthopantomogramme, la VS, l'immunoélectrophorèse des protides et le myélogramme sont les éléments de surveillance.

\section{Conclusion}

Le plasmocytome osseux solitaire de la mandibule est une tumeur rare. La confirmation de son diagnostic est histologique, mais un bilan complet est nécessaire afin d'éliminer un myélome multiple. La radiothérapie constitue le traitement de référence. Le taux de contrôle local semble dépendre de la dose de radiothérapie, qui doit être au minimum de 45 Gy. L'évolution fréquente vers un myélome multiple impose une surveillance étroite et durable de ces patients.

\section{Conflits d'intérêt : aucun}

\section{Références}

1. Tournier-Rangeard L, Lapeyre $M$, Graff-Caillaud $P$, Mege $A$, Dolivet $G$, Toussaint B, et al. Radiotherapy for solitary extramedullary plasmacytoma in the head-and-neck region: A dose greater than $45 \mathrm{~Gy}$ to the target volume improves the local control. Int J Radiat Oncol Biol Phys 2006;64:1013-1017.

2. Gonzales J, Elizondo J, Trull JM, De Torres I. Plasma-cell tumors of the condyle. Br J Oral Maxillofac Surg 1991;29:274-276.

3. Millesi W, Enislidis G, Lindner A, Schobel G, Ewers R, Drach J, Rath T. Solitary plasmocytoma of the mandible: A combined approach for treatment and reconstruction. Int J Oral Maxillofac Surg 1997;26:295-298.

4. Bataille R, Sany J, Serre H. Plasmocytomes apparemment solitaires des os: aspects cliniques et pronostiques. Nouv Presse Med 1981;10:407-411.
5. Ilankovan V, Moos KF, El Attar A. Intramedullary plasma cell tumors. Int J Oral Maxillofac Surg 1990;19:323-326.

6. Florencio M, Jose L, Gil-Diez, Campano FJ, Jose R, Del Hoyo A. Mandibular lesion as the first evidence of multiple myeloma. Case report. J Craniomaxillofac Surg 1989;17:315-317.

7. Shoemaker EI, Romano AJ, Gado M, Hodges FJ. Neuroradiology case of the day: Plasmocytoma of the skull vault. Am J Roentgenol 1989;152:1333-1338.

8. Collangettes-Peyrat D, Baudet-Pommel M, Fonck Y, Meyniel P. Plasmocytome solitaire de la mandibule : étude d'un cas. Actual Odonto Stomatol 1990;169:175-183.

9. Mustoe TH, Fried MP, Goodman ML, Kelly JH, Strome M. Osteosclerotic plasmocytoma of maxillary bone (orbital floor). J Laryngol Otol 1984;98:929-938.

10. Khochtali H, Yacoubi MT, Bouzaiene M, Abassi-Bakir D, Ben Hammouda M, Krifa H. Plasmocytome solitaire : À propos d'un cas de localisation cranio-faciale. Rev Stomatol Chir Maxillofac 1992;93:377-380.

11. Bencheikh R, Benhammou A, Rabeh G, Benbouzid MA, Boulaich M, Essakali L, et al. Plasmocytome solitaire osseux de la mandibule. Rev Stomatol Chir Maxillofac 2007;108:135-138.

12. Mendenhall W, Mendenhall C, Mendenhall N. Solitary plasmacytoma of bone and soft tissues. Am J Otolaryngol 2003;24: 395-399.

13. Liebross RH, Ha CS, Cox JD, Weber D, Delasalle K, Alexanian R. Solitary bone plasmocytoma: Outcome and prognostic factors following radiotherapy. Int J Radiat Oncol Biol Phys 1998;41: 1063-1067.

14. Meyer JE, Schulz MD. Solitary myeloma of bone: Review of 12 cases. Cancer 1974;34:438-440.

15. Ben Ammar CN, Ghorbel I, Kochbati L, Gargouri W, Touati S, Maalej M. Plasmocytome solitaire extramédullaire de la tête et du cou : à propos de cinq cas. Cancer/Radiothérapie 2010;14:755-758.

16. Avilès A, Huerta-Guzman J, Delgado S, Fernandez A, Diaz-Maqueo JC. Improved outcome in solitary bone plasmacytoma with combined therapy. Hematol Oncol 1996;14:111-117.

17. Kochbati L, Ben Romdhane NK, Mrad K, Nasr C, Ben Salah DE, Ben Romdhane K, et al. Plasmocytome solitaire osseux : aspects thérapeutiques et évolutifs. Cancer Radiother 2004;8:70-74. 